\title{
Orbital alveolar soft part sarcoma: A report of 8 cases and review of the literature
}

\author{
YAN HEI $^{*}$, LI KANG $^{*}$, XINJI YANG, YI WANG, XIAOZHONG LU, YUEYUE LI, WEI ZHANG and LIHUA XIAO \\ Institute of Orbital Disease, General Hospital of Chinese People's Armed Police Forces, Beijing 100039, P.R. China
}

Received November 18, 2016; Accepted August 23, 2017

DOI: $10.3892 / 01.2017 .7286$

\begin{abstract}
Alveolar soft part sarcoma (ASPS) is a rare soft tissue neoplasm. The incidence of orbital involvement is the highest in ASPS arising in the head and neck region. However, limited information is available regarding its clinical characteristics. The present study presents the clinical manifestations, imaging findings, pathological features, treatment strategies and prognosis records of 8 cases of orbital ASPS over the last 15 years in a single hospital, along with a review of the literature. Included were 3 male and 5 female patients, with the median age at presentation being 9.5 years. The mean average largest tumour diameter was $3.6 \mathrm{~cm}$. A total of 5 patients underwent surgical excision of the tumour, with 2 undergoing orbital exenteration and 1 undergoing partial orbital exenteration. In total, 6 patients received postoperative radiotherapy and 2 received chemotherapy. Upon follow-up, 6 patients were doing well with no evidence of recurrence or metastasis. Local recurrence developed in 2 patients, of whom 1 succumbed following withdrawal from treatment. According to the present series and the cases mentioned in the literature, orbital alveolar soft part sarcoma has characteristics distinct from those of alveolar soft part sarcoma which arises in other locations. Orbital alveolar soft part sarcoma presents itself in a younger population with a shorter course of disease, smaller tumour size, improved prognosis, a marked association with
\end{abstract}

Correspondence to: Dr Li Kang, Institute of Orbital Disease, General Hospital of Chinese People's Armed Police Forces, 69 Yongding Road, Beijing 100039, P.R. China

E-mail: kangli95@163.com

*Contributed equally

Abbreviations: ASPS, alveolar soft part sarcoma; CT, computed tomography; MRI, magnetic resonance imaging; PAS, periodic acid-Schiff; SMA, smooth muscle actin; TFE3, transcription factor for immunoglobulin heavy-chain enhancer 3; EMA, epithelial membrane antigen; CEA, carcinoembryonic antigen; NSE, neuron-specific enolase; AFP, $\alpha$-fetoprotein

Key words: alveolar soft part sarcoma, extraocular muscle, orbit, pathology, prognosis the extraocular muscles and with the $\mathrm{Ki}-67$ proliferation index possibly associated with prognosis of the disease.

\section{Introduction}

Alveolar soft part sarcoma (ASPS) is a rare form of soft tissue neoplasm that accounts for between 0.5 and $0.9 \%$ of all soft tissue sarcomas, and between 1 and $15 \%$ of malignant tumours in adults and children, respectively (1). ASPS may occur at any age, with a peak incidence in the third decade of life. ASPS is usually identified in the extremities in adults, and the head and neck region in children, particularly the orbit and tongue. However, it is rare for primary ASPS lesions to form in the orbit. In the present study, the clinical manifestations, imaging results, pathological features, treatment strategies and prognosis of 8 cases of orbital ASPS over the last 15 years were reviewed. Domestic and foreign literature regarding ASPS was also reviewed for further comprehension of this rare type of tumour.

\section{Materials and methods}

Ethics statement. The present retrospective study was approved by the Medical Ethics Committee of the General Hospital of Chinese People's Armed Police Forces (Beijing, China) and conducted in compliance with the tenets of The Declaration of Helsinki. All patients or their parents provided written informed consent.

Patients. Patients with orbital ASPS who were treated over the last 15 years (from January 2000 to April 2015) at the General Hospital of Chinese People's Armed Police Forces were considered in the present study $(n=8)$. A comprehensive retrospective review was performed to evaluate the characteristics of this type of sarcoma. Information regarding the clinical course, including clinical manifestations and treatment, was collected from the patients' medical records. Computed tomography (CT) and magnetic resonance imaging (MRI) results were acquired from the database at the General Hospital of Chinese People's Armed Police Forces. Outcome information was obtained from follow-up data and phone calls to the patients or their parents.

Haematoxylin and eosin (H\&E)-stained slides, periodic acid-Schiff (PAS)-stained slides and immunohistochemical slides were available in all cases. The diagnosis of ASPS 
was confirmed by the review of these slides by experienced pathologists at the General Hospital of Chinese People's Armed Police Forces.

A review of the English literature based on a PubMed search spanning 1963 to 2013 and a review of the Chinese literature based on a China Knowledge National Infrastructure search spanning from 1981 to 2014 were performed, and all cases of orbital ASPS with detailed data were included. Cases were excluded if they were duplicated or their information was too generalized.

\section{Results}

Clinical data and ophthalmological examination. From a total of 8 patients, 3 were male and 5 were female, with age at presentation ranging between 2 and 51 years (median, 9.5 years). Patients $<20$ years of age made up $75.0 \%(n=6)$ of the sample, of which 5 were $<10$ years of age $(62.5 \%)$. The duration of symptoms ranged between 9 days and 1 month. The tumour was located in the right orbit in 3 cases and the left orbit in 5 cases. Clinical manifestations were mainly proptosis and abnormal position of the eye (Fig. 1). Certain individuals also exhibited accompanying eyelid swelling, blepharoptosis, headache, ophthalmodynia, impaired vision, diplopia and subconjunctival vasodilatation. Detailed data are presented in Table I.

Table II summarizes the cases of orbital ASPS reported in the literature listed on PubMed (1-22), excluding this series. There were 55 cases ( 33 female and 22 male patients) between 1963 and 2014, with a median age at diagnosis of 12 years (ranging between 10 months and 69 years). The lesion was located in the right orbit in 19 cases, the left orbit in 22 cases and the location of the mass was not provided in 14 cases. The median tumour size was $2.95 \mathrm{~cm}$ in the largest diameter. Table III summarizes the cases reported in the Chinese literature from 1981 to 2014 (23-36). There were 9 female and 10 male patients, totalling 19 cases, with a median age at diagnosis of 11 years (range, 2-53 years). The lesion was located in the right orbit in 7 cases and in the left orbit in 12 cases. The median tumour size was $2.45 \mathrm{~cm}$. When summing up the data in Tables I-III, there were 82 patients with orbital ASPS (47 female and 35 male patients), with a median age of 12 years at diagnosis. The median tumour size was $2.85 \mathrm{~cm}$. There was a slight female predilection (female/male ratio, 1.34:1) in orbital ASPS when compared with ASPS arising in other locations.

Imaging features. Ultrasonography was performed in 4 of the selected cases, which demonstrated hypoechoic inhomogeneous soft tissue masses with increased flow (Fig. 2). A computed tomography (CT) examination revealed well-circumscribed ovoid and enhanced masses in all cases (Fig. 3A). MRI demonstrated that all the tumours exhibited intermediate signal intensity in T1- and T2-weighted images (Fig. 3B and C), in addition to marked enhancement in contrast-enhanced images (Fig. 3D). Imaging features demonstrated that the lesions were located in the medial part of the orbit in 3 cases, in the supraorbital foramen in 2 cases and in the infraorbital foramen in 2 cases, and, in 1 case, the lesion filled the orbital cavity. The tumours were associated with the extraocular muscles (Figs. 2D and 3E-H). Tumours were located intramuscularly in 3 cases, indistinguishable from the muscles in 3 cases and abutting the muscles in 2 cases. The average largest diameter of the masses was $3.6 \mathrm{~cm}$ (range, $2.0-5.0 \mathrm{~cm})$.

Histopathological features. As presented in Fig. 4, overall, these poorly circumscribed pale grey or grey-yellow masses were soft and friable, tending to haemorrhage (Fig. 4A). Microscopic examination identified that the characteristic arrangement of the tumour cells was organoid, in a nesting or alveolar pattern, which was more obvious at low magnification. The size and appearance of the nests tended to be uniform. The tumour cell nests were separated by delicate septa of connective tissue containing sinusoidal vascular channels lined by flattened endotheliocytes. The large oval or polygonal tumour cells had a distinctly epithelioid appearance with somewhat similar size and shape. The cell borders were sharply defined. There were 1 or 2 large vesicular nuclei with prominent nucleoli per cell. Nuclear atypia was not common. Eosinophilic cytoplasm was abundant, with a finely granular, transparent or vacuolar pattern (Fig. 4C). Mitotic figures were uncommon. All cases were positive for diastase following PAS staining (Fig. 4D), although this may not have been apparent from H\&E staining. Tumour emboli could be observed in the small vessels in the tumour tissue of cases 4 and 5. Cases 2, 3 and 4 were positive for vimentin by immunohistochemistry. Cases 1, 6 and 7 had desmin-positive foci (Fig. 4F). Smooth muscle actin was positive in a number of tumour cells in case 6 . Transcription factor binding to immunoglobulin heavy-chain enhancer 3 (TFE3) was positive in all cases with the exception of case 3 (Fig. 4G). Cluster of differentiation (CD) 147 was positive in all cases. Other markers, including cytokeratin, epithelial membrane antigen (EMA), carcinoembryonic antigen, actin, neuron-specific enolase (NSE), synaptophysin, chromogranin A, S-100 protein, melanoma, melan A, CD99, CD34 and $\alpha$-fetoprotein, were negative in all cases. The antigen Ki-67 proliferation index was $<10 \%$ in the majority of cases, but was $15 \%$ in cases 2 and 3 (Fig. 4H; Table IV).

Treatment and follow-up. Cases 1, 3, 4, 5 and 8 underwent surgical excision of the tumour, cases 2 and 6 underwent orbital exenteration, and case 7 underwent partial orbital exenteration. Cases 1, 2, 4, 5, 6 and 8 received postoperative local radiotherapy on the orbital region. Cases 6 and 8 received chemotherapy. Case 1 was doing well at 6 months and was then lost to follow-up. Cases $4,5,6,7$ and 8 were doing well with no evidence of local recurrence or metastasis during the follow-up period (between 3 and 61 months). Local recurrence of the tumour developed at 3 months post-tumorectomy in case 3 , with the patient succumbing 1 year after being withdrawn from treatment by the patient's parents. Case 2 visited the hospital presenting with recurrence of the tumour 3 months after undergoing tumorectomy at another hospital. A doctor advised orbital exenteration, which the patient declined, instead opting to undergo excision of the tumour only. Local recurrence developed again after 3 months. CT and MRI demonstrated that the orbital cavity was occupied by the tumour. The patient underwent orbital exenteration. The tumour was recurrent 3 months after surgery and the patient received radiotherapy. The patient complained of gradually aggravated headaches after 


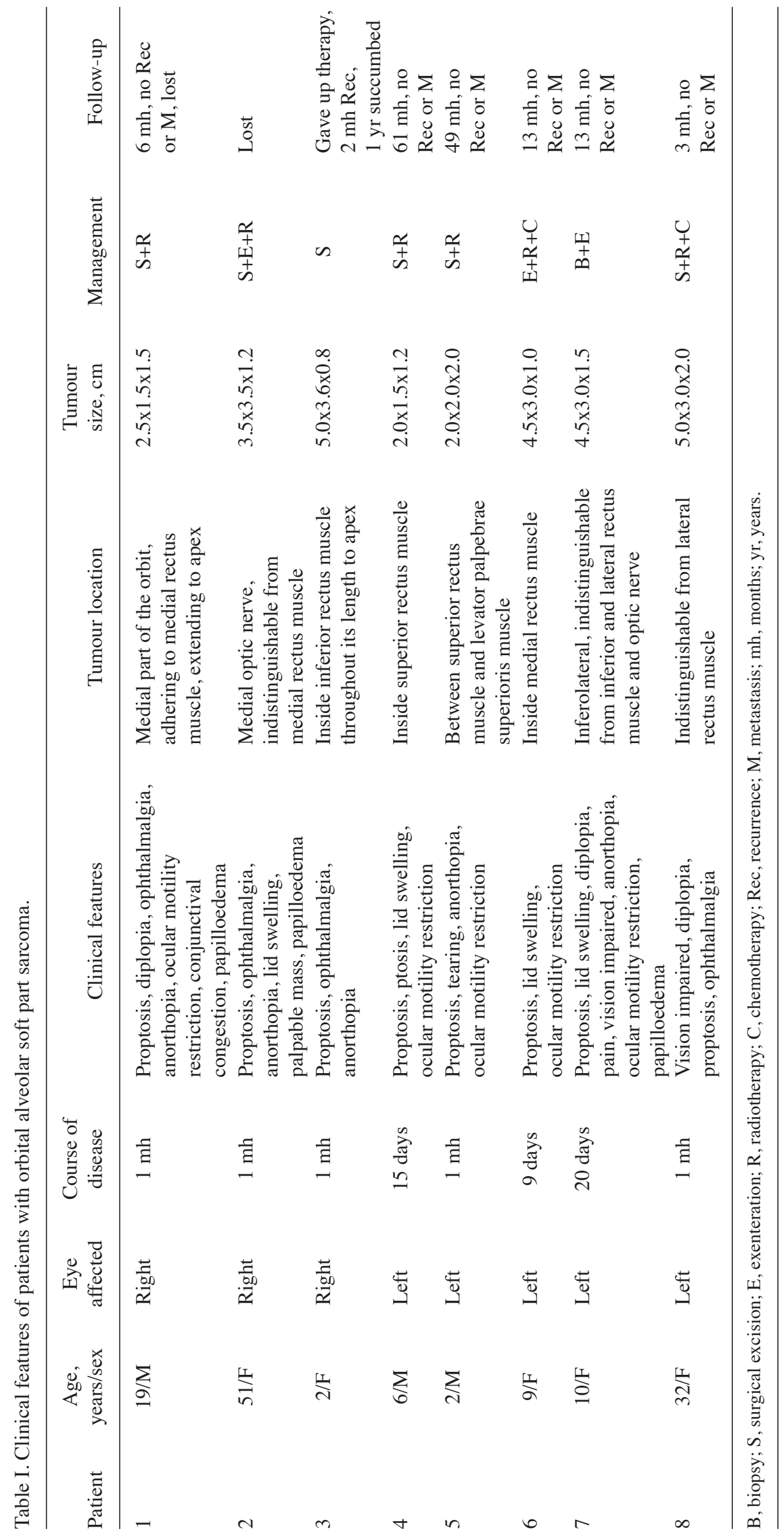




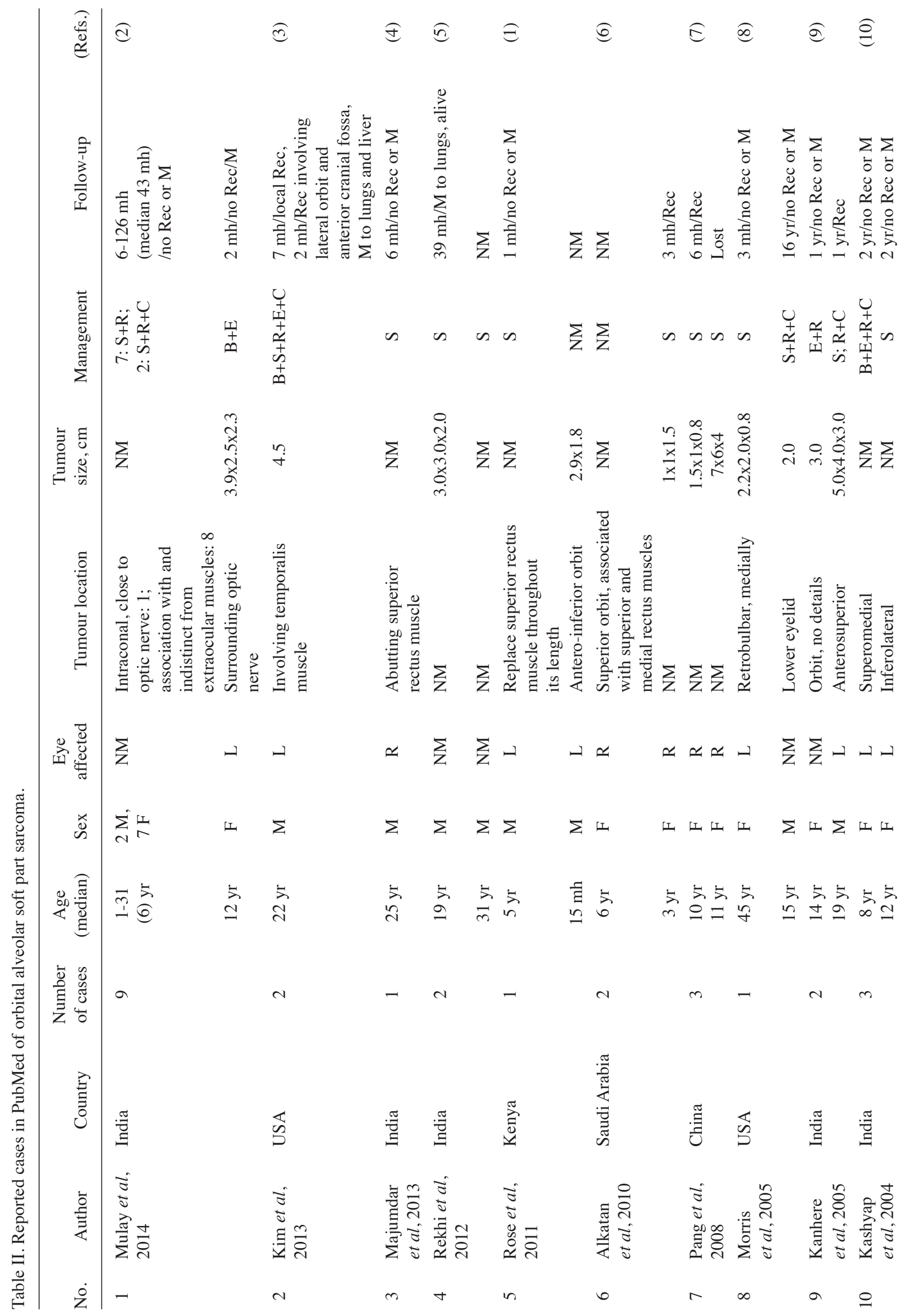




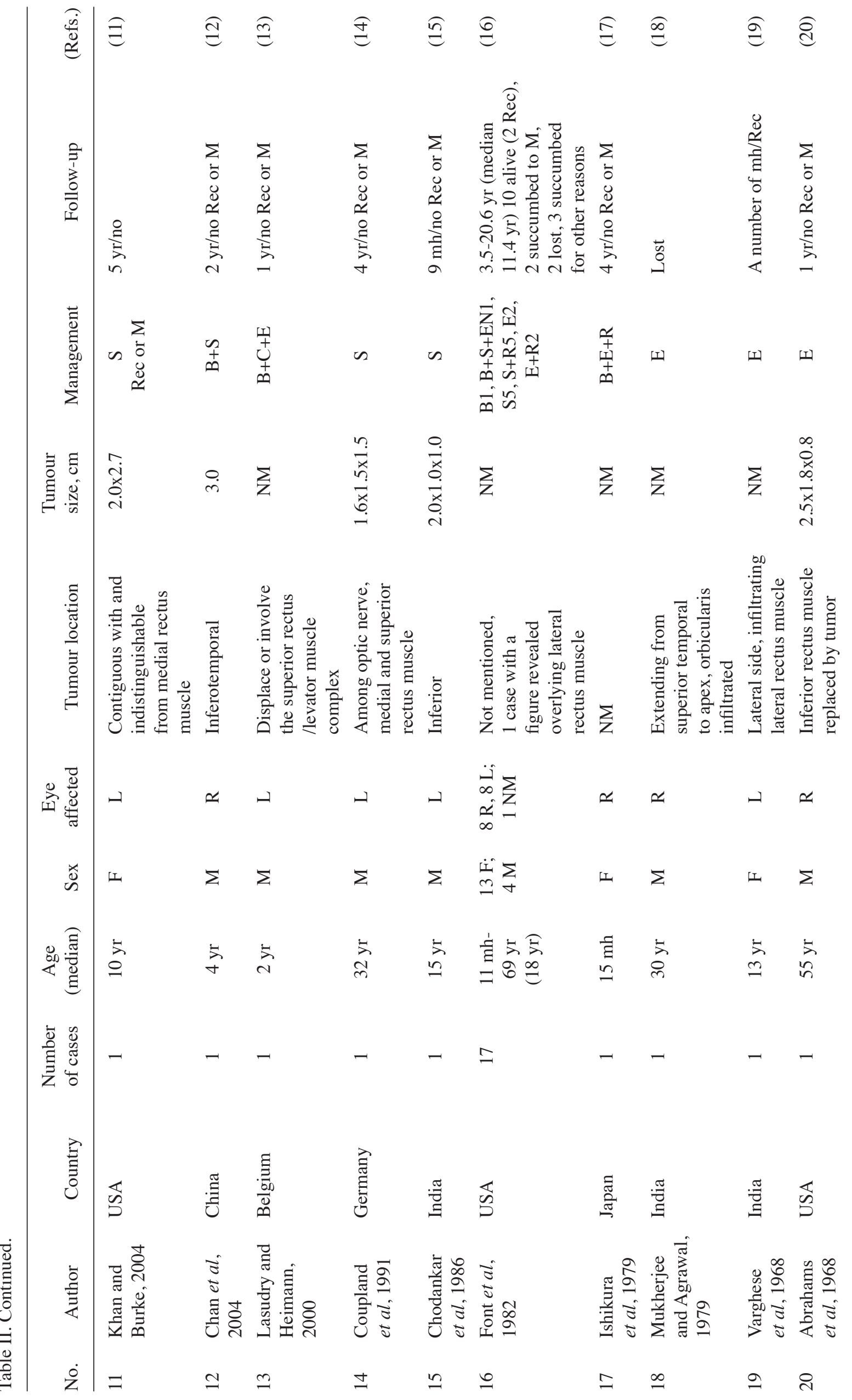


1 month. MRI demonstrated invasion to the sphenoid bone. The patient eventually succumbed.

The median period of follow-up following surgical treatment was 12 months (range, 3-61 months) in the series of the present study, whereas the period was 12 months (range, 2-247 months) in the PubMed literature (Table II) and 48 months (range, 2-108 months) in the Chinese literature (Table III). In the 65 patients with follow-up data, 43 (66.2\%) were alive with no evidence of disease during the follow-up period, 12 patients (18.5\%) were alive with local recurrence, and $2(3.1 \%)$ exhibited metastases to the lungs and liver. Certain patients $(n=4 ; 6.2 \%)$ succumbed to metastatic disease and $4(6.2 \%)$ succumbed to non-related causes.

\section{Discussion}

Alveolar soft part sarcoma (ASPS) is a rare tumour primarily affecting children and adolescents. There is a slight female preponderance in adults (60\% of patients are female) (37), which is not observed in children (38). ASPS predominantly affects the deep soft tissues of the extremities in adults, particularly the thighs and buttocks; the arms, thorax and retroperitoneum are seldom involved (39). The most common location in children is the head and neck region, particularly the orbit and tongue $(16,40)$. Following the review of hundreds of reports, it was identified that, in 172 cases, orbital involvement had the second-highest incidence (15.1\%), following the buttock and thigh, and that orbital involvement had the highest incidence in ASPS arising in the head and neck region $(55 \%)(41)$.

Characteristics of orbital ASPS. From these data, it was concluded that orbital ASPS has characteristics distinct from those of ASPS arising in other locations: i) Orbital ASPS involves a younger population; the median age of the patients when ASPS is diagnosed in locations other than the orbit was $>30$ years $(42,43)$, whereas that of patients with orbital ASPS is $<15$ years; ii) the course of disease is usually shorter in orbital ASPS since the symptoms are easily noticed by the patient or parents, therefore: i) Tumour size in orbital ASPS is frequently smaller than ASPS arising in other locations. The median size of orbital ASPS is usually $<5 \mathrm{~cm}$, whereas that in other locations is $>5 \mathrm{~cm}(5,42)$.

Clinical features. Orbital ASPS has no defining clinical features. As with other space-occupying lesions in the orbit, patients with ASPS primarily present with proptosis, abnormal position of the eyes, eyelid swelling, and conjunctival congestion with dilated and tortuous vessels (16). In certain cases, metastasis to the lungs or brain may be the presenting feature of ASPS (44).

Imaging features. Ultrasonography of ASPS is non-specific, but often reveals a slightly hypoechoic or hyperechoic and heterogeneous soft tissue mass with markedly increased blood flow. CT usually demonstrates a soft tissue mass that is homogeneous and isodense to muscle with vigorous enhancement. Reports in the literature on MRI of this tumour have identified intermediate or hyperintense signal intensity in T1-weighted images and hyperintense signal intensity in $\mathrm{T} 2$-weighted images. 


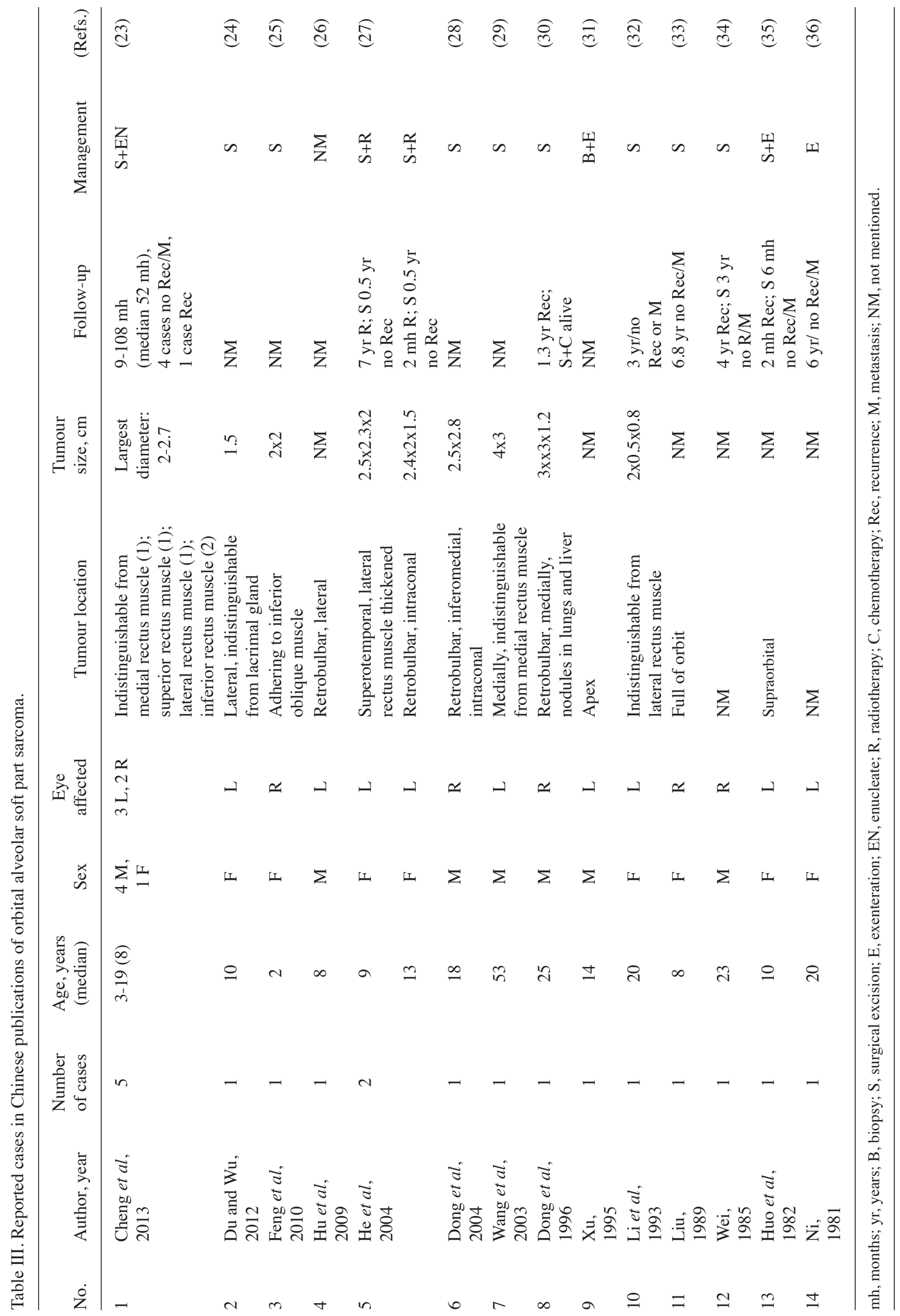


Table IV. Immunohistochemical results of the patients with orbital alveolar soft part sarcoma.

\begin{tabular}{|c|c|}
\hline Case & Immunohistochemical result \\
\hline 1 & Vimentin $^{-}$desmin focus ${ }^{+}$actin $^{-} \mathrm{CK}^{-} \mathrm{NSE}^{-} \mathrm{Syn}^{-} \mathrm{CgA}^{-} \mathrm{S}^{-} 100^{-} \mathrm{TFE}^{++} \mathrm{CD}^{-147^{+}} \mathrm{Ki}^{-} 67+5 \%$ \\
\hline 2 & Vimentin focus ${ }^{+}$desmin $^{-} \mathrm{CK}^{-} \mathrm{NSE}^{-} \mathrm{Syn} \mathrm{CgA}^{-} \mathrm{S}-100^{-} \mathrm{CEA}^{-} \mathrm{TFE}^{++} \mathrm{CD} 147^{+} \mathrm{Ki}-67+15 \%$ \\
\hline 3 & 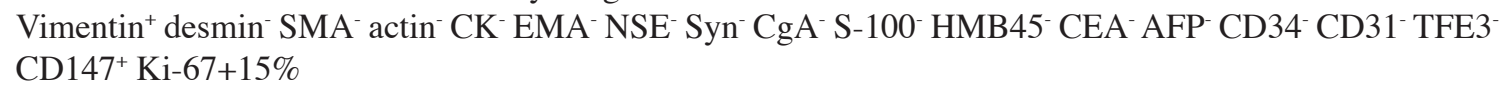 \\
\hline 4 & Vimentin $^{+}$desmin ${ }^{-}$actin $^{-} \mathrm{CK}^{-} \mathrm{NSE}^{-} \mathrm{Syn}^{-} \mathrm{CgA}^{-} \mathrm{S}-100^{-} \mathrm{HMB}^{-} 5^{-} \mathrm{AFP}^{-} \mathrm{CD} 34^{-} \mathrm{CD} 99^{-} \mathrm{TFE}^{++}{ }^{++} \mathrm{CD} 147^{+} \mathrm{Ki}-67+5 \%$ \\
\hline 5 & 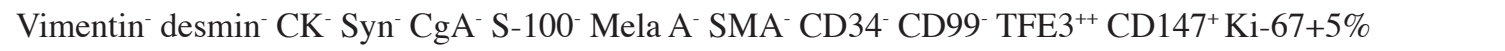 \\
\hline 6 & Vimentin $^{-}$desmin partly ${ }^{+} \mathrm{CK}^{-} \mathrm{NSE}^{-} \mathrm{Syn}^{-} \mathrm{CgA}^{-} \mathrm{S}-100^{-} \mathrm{AFP}^{-} \mathrm{SMA}^{-}$artly ${ }^{+} \mathrm{CD} 34^{++} \mathrm{TFE}^{++} \mathrm{CD}^{+} 147^{+} \mathrm{Ki}-67+10 \%$ \\
\hline 7 & Vimentin ${ }^{-}$desmin partly ${ }^{+} \mathrm{CK}^{-} \mathrm{NSE}^{-} \mathrm{Syn}^{-} \mathrm{CgA}^{-} \mathrm{S}-100^{-} \mathrm{AFP}^{-} \mathrm{SMA}^{-} \mathrm{CD} 34^{++} \mathrm{TFE}^{+++} \mathrm{CD}^{+4} 7^{+} \mathrm{Ki}-67+8 \%$ \\
\hline 8 & 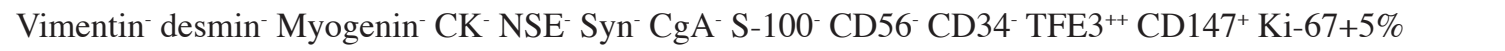 \\
\hline
\end{tabular}

CK, cytokeratin; NSE, neuron-specific enolase; Syn, synaptophysin; CgA, chromogranin A; TFE3, transcription factor for immunoglobulin heavy-chain enhancer 3; CD147, cluster of differentiation 147.

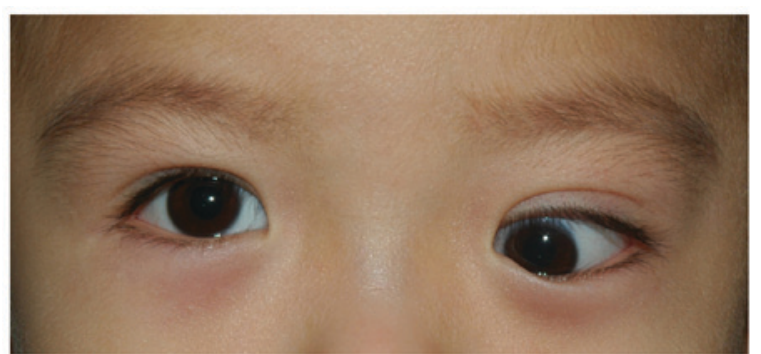

Figure 1. Clinical image of case 5 demonstrating non-axial proptosis of the left eye with downward and rightward displacement of the globe.
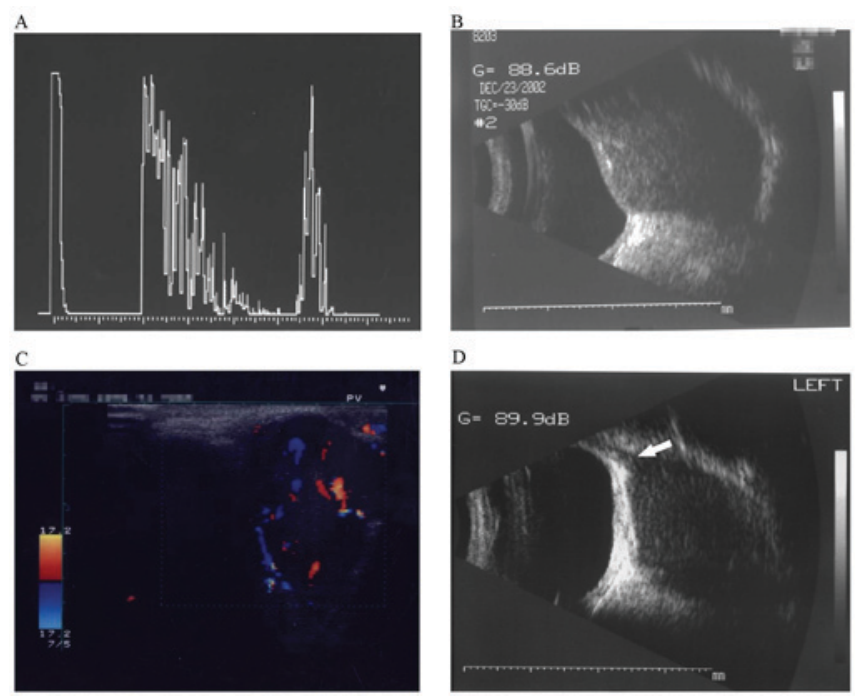

Figure 2. Ultrasonography imaging of alveolar soft part sarcoma. (A) A-scan ultrasonography of case 2 demonstrated low to moderate internal reflectivity with a high velocity peak. (B) B-scan ultrasonography of case 2 revealed a hypoechoic, homogeneous soft tissue mass indenting the globe. (C) Colour Doppler flow imaging of case 2 revealed marked increased blood flow within the mass. (D) B-scan ultrasonography of case 1 revealed a hypoechoic, homogeneous soft tissue mass. The arrow indicates the attachment point of the medial rectus muscle. The mass had an association with the medial rectus muscle.

The majority of lesions exhibit marked intense enhancement in contrast-enhanced MRI $(45,46)$. A number of studies have focused on the characteristic imaging features of orbital ASPS. In the present series of cases analysed, all the lesions demonstrated intermediate signal intensity in T1- and T2-weighted images, the majority with marked enhancement in contrast-enhanced images.

Diagnosis and differential diagnosis. There are no distinct characteristics in the clinical and imaging features of this kind of tumour. Owing to the high vascularization, the tumour may be misinterpreted as a type of vasogenic tumour, including hemangioma, lymphangioma or hemangiopericytoma, by clinicians prior to surgery (47). The final diagnosis should be dependent on the pathological examination following surgery. The pathomorphological features of ASPS should allow for differential diagnosis from other tumours, including paraganglioncytoma, granular cell tumour, amelanotic melanoma, alveolar rhabdomyosarcoma and metastatic renal cell carcinoma (48). PAS is an important stain for the diagnosis of ASPS, as ASPS possesses PAS-positive diastase-resistant granules in $>80 \%$ of cases (49). All the present cases were PAS-positive. Immunohistochemical examination is also important for differential diagnosis. The majority of ASPS tumour cells exhibit moderate to strong nuclear staining of TFE3, which is a sensitive and specific marker for ASPS. Monocarboxylate transporter protein 1 and its cellular chaperone protein CD147 also demonstrate positive reactions in PAS-positive diastase-resistant cells. There are no other specific markers for ASPS, which does not express the common antigens of the epithelium (e.g. cytokeratin and EMA), neuroendocrine cells (e.g. NSE, synaptophysin, chromogranin and S-100 protein) or melanocytes (e.g. human melanoma black- 45 and melan A). However, certain markers are positive in the aforementioned tumours, allowing for differential diagnosis. The representative pathological features combined with supplementary examinations, including immunohistochemical staining, PAS staining and molecular biology technologies, are useful for the pathological and differential diagnosis of ASPS.

Histogenesis of the tumour. The histogenesis of ASPS has been controversial for a number of years. Genetic studies on ASPS have confirmed a specific fusion gene involving 

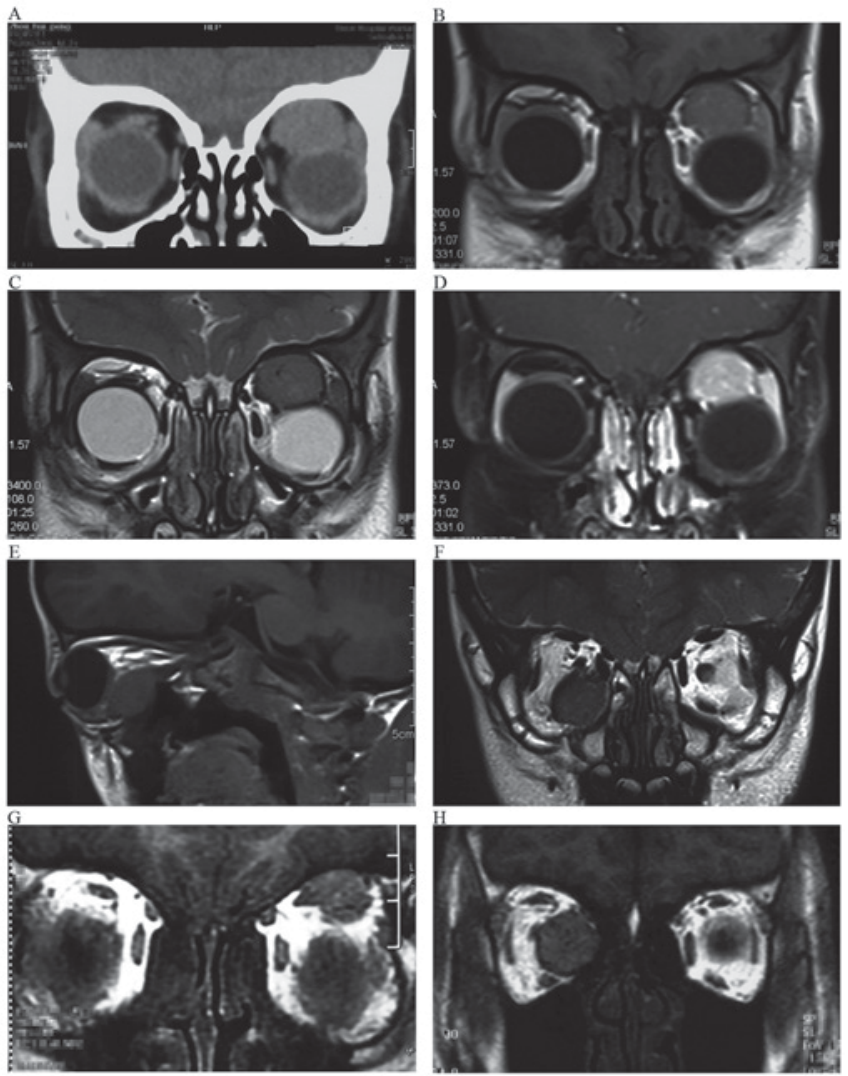

Figure 3. CT and MRIs of alveolar soft part sarcoma. (A) Coronal section of CT imaging of case 5 showing a well-defined homogeneous mass with equal density to muscle in the left orbit. It was indistinguishable from the superior rectus/levator muscle complex with marked downward displacement of the globe. (B) Coronal T1-weighted MRI of case 5 revealed an ovoid isointense mass in the left medial superior orbit that was indistinguishable from the superior rectus/levator muscle complex with marked downward displacement of the globe. (C) Coronal T2-weighted MRI of case 5 revealed an ovoid isointense mass in the left medial superior orbit. (D) Coronal contrast-enhanced and fat-suppressed T1-weighted MRI of case 5 revealed an ovoid mass with marked enhancement in the left medial superior orbit. (E) Axial T1-weighted MRI of case 3 revealed an ovoid isointense mass in the inferior part of the right orbit indistinguishable from the inferior rectus muscle throughout its length to the apex with marked upward displacemen of the globe. (F) Coronal T2-weighted MRI of case 3 revealed an ovoid isointense mass in the inter-inferior part of the right orbit indistinguishable from the inferior rectus muscle with marked out-upward displacement of the globe. (G) Coronal T1-weighted MRI of case 4 revealed an ovoid isointense mass in the left superior orbit indistinguishable from the superior rectus muscle with marked downward displacement of the globe. $(\mathrm{H})$ Coronal T1-weighted MRI of case 1 revealed an ovoid isointense mass in the medial part of the right orbit indistinguishable from the medial rectus muscle with marked outward displacement of the globe. CT, Computed tomography; MRI, magnetic resonance imaging.

the ASPS critical region-1 gene and TFE3 and the resultant fusion protein generated by the unbalanced chromosomal translocation [der(17)t(X:17)(p11;25)] (50). Therefore, it is generally accepted that ASPS is a sarcoma associated with chromosomal translocation, therefore it is sorted into a class containing uncertain differentiated tumours.

It was hypothesized for a number of years that ASPS represented an unusual form of myogenic tumour (51-53). Subsequently, conflicting results on the expression of muscle-associated markers have disproved this hypothesis $(54,55)$. Previously, genetic expression profiles of ASPS identified that certain muscle-specific transcripts were
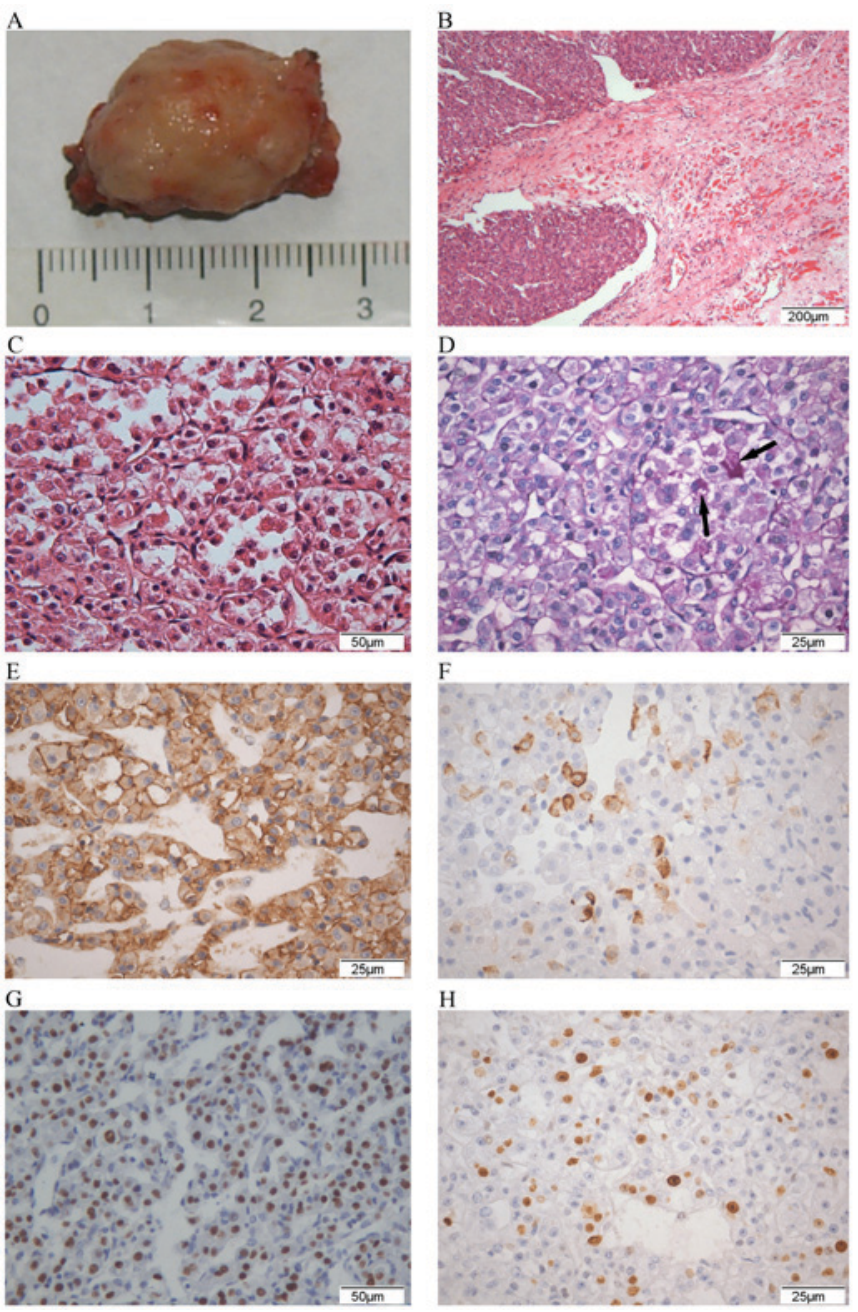

Figure 4. Histopathological examination of alveolar soft part sarcoma (A) Gross examination of the histopathological specimen revealed an encapsulated mass measuring $2.5 \times 1.5 \times 1.5 \mathrm{~cm}$. It had adhered to the medial rectus muscle of the right eye and extended to the apex. (B) Image of tissue from case 7 showing the association between the tumour and the extraocular muscle. The left top of the fig. is the tumour and the remaining muscle is located at the low right (H\&E; magnification, $\mathrm{x} 50$ ). (C) Image of tissue from case 5 demonstrating nesting or organoid arrangement separated by delicate partitions of fibrous connective tissue, which formed a characteristic pseudoalveolar pattern. Blood sinusoid was easily observed between the nests. The individual tumour cells were round or polygonal with abundant eosinophilic and finely granular cytoplasm and those at the nest centre were sparse. Nuclear atypia was not obvious (H\&E; magnification, $\mathrm{x} 200$ ). (D) PAS stain with diastase digestion of tissue from case 7 exhibited the characteristic PAS-positive pruinose crystalline inclusions in the cytoplasm of certain tumour cells (indicated by arrow). (PAS; magnification, $x 400$ ). (E) Image of tissue from case 6 showing strong membrane immunoreactivity of CD147 in certain tumour cells (DAB; magnification, x400). (F) Desmin immunostaining identified focal cytoplasmic immunoreactivity in tissue from case 7 (DAB; magnification, x400). (G) The transcription factor for immunoglobulin heavy-chain enhancer 3 immunostaining of tissue from case 6 demonstrated specific marked nuclear immunoreactivity in certain tumour cells (DAB; magnification, $\mathrm{x} 200$ ). (H) Immunostaining of tissue from case 3 revealed nuclear immunoreactivity for Ki-67 in certain tumour cells (DAB; magnification, x400). PAS, periodic acid-Schiff; CD, cluster of differentiation; DAB, 3,3'-diaminobenzidine; H\&E, haematoxylin and eosin.

increased, which suggests an association with muscle cell progenitors (56). ASPS has a marked predilection to arise on the thighs, buttocks and abdominal or chest walls, which suggests that ASPS is associated with skeletal muscles or 
musculofascial planes (37). The present series and the cases reviewed revealed that orbital ASPS is generally associated with the extraocular muscles. There were 19 and 9 patients in Tables II and III respectively, whose tumours had been mentioned definitely to be abutting, adhering to or indistinguishable from the extraocular muscles. In the present series, the tumours were clearly observed during the surgical procedures. The tumours were revealed following longitudinally splitting the thickened extraocular muscles in 3 cases, whereas the tumours grew along with and were indistinguishable from the extraocular muscles in 3 cases. There were 6 cases expressing vimentin or desmin in the present series, which were identified using immunohistochemistry. Although immunohistochemical examination of muscle-associated markers in previous years did not demonstrate myogenic histogenesis, the growth pattern of this tumour suggests that orbital ASPS is associated with the extraocular muscles.

Treatment. Generally, complete surgical excision of the localized tumour with a microscopically negative margin is the established treatment for ASPS. The possibility of recurrence is rare if the lesion has been completely resected (57). The pathological status of surgical margin, which indicates whether active tumour tissue remains, is important for prognosis. Surgery is also the primary treatment in orbital ASPS; however, due to the narrow space and complex organization of the orbit, it is occasionally impossible to perform a complete resection if the patient declines to undergo an exenteration. Previous studies have demonstrated that chemotherapy and radiotherapy do not improve the survival rate; however, radiotherapy as an adjuvant therapy has been demonstrated to decrease the risk of local recurrence $(50,58)$. Metastasectomy may markedly prolong the survival rate of patients with pulmonary metastases of ASPS $(16,37)$.

Prognosis. It has been reported that the biological behaviour of ASPS is relatively indolent, with the 2-, 5-, 10- and 20-year overall survival rates being $77,60,38$ and $15 \%$, respectively (37). Metastases occur most commonly in the lungs, brain and bones (37). Since $\sim 1 / 3$ patients develop metastases to the lungs or brain decades after initial diagnosis, long-term follow-up is strongly advised for all cases of ASPS.

According to previous studies, the patient's age, tumour size and presence of metastases at diagnosis are significant prognostic factors in ASPS. It is hypothesized that younger age with a tumour size of $<5 \mathrm{~cm}$ and absence of metastases is associated with a more favourable outcome $(48,59)$. If the tumour is resected completely, younger patients may achieve a good prognosis with prolonged survival rates $(40,60)$. Certain studies have reported $100 \%$ survival rates beyond 5 years in paediatric patients (38). However, there was an exception in the present series: A 2-year-old patient succumbed 1 year after diagnosis, possibly due to the large tumour and withdrawal from therapy.

The prognosis is improved in patients with ASPS located in the orbit and tongue, which is possibly associated with smaller lesions, younger age at diagnosis and shorter course of disease $(16,40)$, which was also demonstrated in the present review.
It is known that there are no histopathological features predictive of prognosis in ASPS (48). However, in the present series, 2 cases (cases 2 and 3 ) recurred shortly following surgery ( $<3$ months). Of these, 1 patient succumbed 1 year later and the other had an invasion to the brain that was possibly fatal. It is notable that the $\mathrm{Ki}-67$ proliferation index was $15 \%$ in these 2 cases, whereas it was $\leq 10 \%$ in 5 cases, suggesting that the Ki-67 proliferation index may be associated with ASPS prognosis. Further study is required in order to investigate this association.

In conclusion, orbital ASPS exhibits characteristics that are distinct from those of ASPS arising in other locations. Orbital ASPS usually affects a younger population, the course of disease is shorter and the tumour size is smaller. Therefore, the prognosis is improved in orbital ASPS. Orbital ASPS may have an association with the extraocular muscles and the Ki-67 proliferation index may be associated with prognosis of ASPS.

\section{References}

1. Rose AM, Kabiru J and Rose GE: Alveolar soft-part sarcoma of the orbit. Afr J Paediatr Surg 8: 82-84, 2011.

2. Mulay K, Ali MJ, Honavar SG and Reddy VA: Orbital alveolar soft-part sarcoma: Clinico-pathological profiles, management and outcomes. J Cancer Res Ther 10: 294-298, 2014.

3. Kim HJ, Wojno T, Grossniklaus HE and Shehata BM: Alveolar soft-part sarcoma of the orbit: Report of 2 cases with review of the literature. Ophthal Plast Reconstr Surg 29: e138-e142, 2013.

4. Majumdar K, Saran R, Tyagi I, Jain A, Jagetia A, Sinha S and Singh A: Cytodiagnosis of alveolar soft part sarcoma: Report of two cases with special emphasis on the first orbital lesion diagnosed by aspiration cytology. J Cytol 30: 58-61, 2013.

5. Rekhi $\mathrm{B}$, Ingle $\mathrm{A}$, Agarwal $\mathrm{M}$, Puri $\mathrm{A}$, Laskar $\mathrm{S}$ and Jambhekar NA: Alveolar soft part sarcoma 'revisited': Clinicopathological review of 47 cases from a tertiary cancer referral centre, including immunohistochemical expression of TFE3 in 22 cases and 21 other tumors. Pathology 44: 11-17, 2012.

6. Alkatan H, Al-Shedoukhy AA, Chaudhry IA and Al-Ayoubi A: Orbital alveolar soft part sarcoma: Histopathologic report of two cases. Saudi J Ophthalmol 24: 57-61, 2010.

7. Pang LJ, Chang B, Zou H, Qi Y, Jiang JF, Li HA, Hu WH, Chen YZ, Liu CX, Zhang WJ and Li F: Alveolar soft part sarcoma: A biomarker diagnostic strategy using TFE3 immunoassay and ASPL-TFE3 fusion transcripts in paraffin-embedded tumor tissues. Diagn Mol Pathol 17: 245-252, 2008.

8. Morris WR, Padgett DM, Osborn FD and Fleming JC: Pathologic quiz case: An orbital mass in a 45-year-old woman. Alveolar soft part sarcoma. Arch Pathol Lab Med 129: 534-536, 2005.

9. Kanhere HA, Pai PS, Neeli SI, Kantharia R, Saoji RR and D'cruz AK: Alveolar soft part sarcoma of the head and neck. Int J Oral Maxillofac Surg 34: 268-272, 2005.

10. Kashyap S, Sen S, Sharma MC, Betharia SM and Bajaj MS: Alveolar soft-part sarcoma of the orbit: Report of three cases. Can J Ophthalmol 39: 552-556, 2004.

11. Khan AO and Burke MJ: Alveolar soft-part sarcoma of the orbit. J Pediatr Ophthalmol Strabismus 41: 245-246, 2004.

12. Chan WM, Liu DT, Lai CK, Tse MW, Ng JS and Lam DS: Soft tissue sarcomas. Case 2. Orbital alveolar soft part sarcoma in a child. J Clin Oncol 22: 2027-2029, 2004.

13. Lasudry J and Heimann P: Cytogenetic analysis of rare orbital tumors: Further evidence for diagnostic implication. Orbit 19: 87-95, 2000.

14. Coupland SE, Heimann H, Hoffmeister B, Lee WR, Foerster $\mathrm{MH}$ and Gross U: Immunohistochemical examination of an orbital alveolar soft part sarcoma. Graefes Arch Clin Exp Ophthalmol 237: 266-272, 1999.

15. Chodankar CM, Pandit SP, Joshi MG and Deodhar KP: Alveolar soft-part sarcoma of the orbit (a case report). Indian J Ophthalmol 34: 67-68, 1986.

16. Font RL, Jurco S III and Zimmerman LE: Alveolar soft-part sarcoma of the orbit: A clinicopathologic analysis of seventeen cases and a review of the literature. Hum Pathol 13: 569-579, 1982 . 
17. Ishikura A, Kimura A and Yamamoto S: A case of alveolar soft part sarcoma. Jpn J Cancer Clin 25: 693, 1979.

18. Mukherjee PK and Agrawal S: Alveolar soft part sarcoma of the orbit. Indian J Ophthalmol 27: 15-17, 1979.

19. Varghese S, Nair B and Joseph TA: Orbital malignant non-chromaffin paraganglioma. Alveolar soft tissue sarcoma. $\mathrm{Br}$ J Ophthalmol 52: 713-715, 1968.

20. Abrahams IW, Fenton RH and Vidone R: Alveolar soft-part sarcoma of the orbit. Arch Ophthalmol 79: 185-188, 1968

21. Altamirano-Dimas M and Albores-Saavedra J: Alveolar soft part sarcoma of the orbit. Arch Ophthalmol 75: 496-499, 1966.

22. Nirankari MS, Greer $\mathrm{CH}$ and Chaddah MR: Malignant non-chromaffin paraganglioma in the orbit. Br J Ophthalmol 47: 357-363, 1963

23. Cheng Y, Sha Y, Huang W, Hong R and Cai R: CT and MRI features of orbital alveolar soft part sarcoma. Chin J Radiol 47: 1147-1148, 2013 (In Chinese).

24. Du C and Wu F: A case report of orbital alveolar soft part sarcoma and review of literatures. J ClinRadiol 31: 1814-1815, 2012 (In Chinese).

25. Feng N, Ma Z and Li C: Ultrasound features of a case of right orbital alveolar soft part sarcoma. Chin J Ultrasonogr 19: 687, 2010 (In Chinese).

26. Hu P, Zhou Z, Wang J, Peng W, Cheng Y, Zhu L and Tang F: Radiological features and clinicopathological findings of alveolar soft part sarcoma. Chin Oncol 19: 12-16, 2009 (In Chinese).

27. He W, Luo Q, Xia R and Yang G: Two cases of orbital relapsing alveolar soft part sarcoma. Chin J Ophthalmol 40: 848-850, 2004 (In Chinese)

28. Dong G, Wang J, and Xie D: A case of intraorbital alveolar soft part sarcoma. Chin J Pract Ophthalmol 22: 320, 2004 (In Chinese)

29. Wang $R$ and Wang W: A case of alveolar soft part sarcoma in orbit, sphenoidal, frontal, ethmoid and maxillary sinus. Chin Arch Otolaryngol Head Neck Surg 10: 279, 2003 (In Chinese).

30. Dong X, Wang L and Yan Q: Intraorbital alveolar soft part sarcoma. Chin J Diagnpathol 3: 184, 1996 (In Chinese).

31. Xu D: A case report of orbital alveolar soft part sarcoma Guangdong Med J 16: 428-429, 1995 (In Chinese).

32. Li M, Zhang D and Yan H: Primary orbital alveolar soft part sarcoma. J Clin Ophthalmol 1: 123-124, 1993 (In Chinese).

33. Liu F: A case of orbital alveolar soft part sarcoma. Chin J Cancer 4: 331, 1989 (In Chinese).

34. Wei Y: A case of orbital alveolar soft part sarcoma. Chin J Ophthalmol 21: 252, 1985 (In Chinese).

35. Huo J, Xiong Q, Ni K and Wang R: Investigation of a case of orbital tumor. J Xinjiang Med University 1: 322, 1982 (In Chinese).

36. Ni C: Observation of ultrastructure of primary orbital alveolar soft part sarcoma. Chin J Ophthalmol 17: 5-7, 1981 (In Chinese).

37. Lieberman PH, Brennan MF, Kimmel M, Erlandson RA Garin-Chesa P and Flehinger BY: Alveolar soft-part sarcoma. A clinico-pathologic study of half a century. Cancer 63: 1-13, 1989.

38. Casanova M, Ferrari A, Bisogno G, Cecchetto G, Basso E, De Bernardi B, Indolfi P, Fossati Bellani F and Carli M: Alveolar soft part sarcoma in children and adolescents: A report from the soft-tissue sarcoma italian cooperative group. Ann Oncol 11: 1445-1449, 2000.

39. Evans HL: Alveolar soft-part sarcoma. A study of 13 typical examples and one with a histologically atypical component Cancer 55: 912-917, 1985

40. Fanburg-Smith JC, Miettinen M, Folpe AL, Weiss SW and Childers EL: Lingual alveolar soft part sarcoma; 14 cases: Novel clinical and morphological observations. Histopathology 45: 526-537, 2004.

41. Ordóñez NG: Alveolar soft part sarcoma: A review and update. Adv Anat Pathol 6: 125-139, 1999.

42. Pennacchioli E, Fiore M,Collini P, Radaelli S, Dileo P, Stacchiotti S, Casali PG and Gronchi A: Alveolar soft part sarcoma: Clinical presentation, treatment, and outcome in a series of 33 patients at a single institution. Ann Surg Oncol 17: 3229-3233, 2010.
43. Reis H, Hager T, Wohlschlaeger J, Bauer S, Katenkamp K, Katenkamp D and Baba HA: Mammalian target of rapamycin pathway activity in alveolar soft part sarcoma. Hum Pathol 44: 2266-2274, 2013

44. Khanna P, Paidas CN and Gilbert-Barness E: Alveolar soft part sarcoma: Clinical, histopathological, molecular, and ultrastructural aspects. Fetal Pediatr Pathol 27: 31-40, 2008.

45. Viry F, Orbach D, Klijanienko J, Fréneaux P, Pierron G, Michon J, Neuenschwander S and Brisse HJ: Alveolar soft part sarcoma-radiologic patterns in children and adolescents. Pediatr Radiol 43: 1174-1181, 2013.

46. Chen YD, Hsieh MS, Yao MS, Lin YH and Chan WP: MRI of alveolar soft part sarcoma. Comput Med Imaging Graph 30: 479-482, 2006

47. Chu WC, Howard RG, Roebuck DJ, Chik KW and Li CK: Periorbital alveolar soft part sarcoma with radiologic features mimicking haemangioma. Med Pediatr Oncol 41: 145-146, 2003.

48. Folpe AL and Deyrup AT: Alveolar soft-part sarcoma: A review and update. J Clin Pathol 59: 1127-1132, 2006.

49. Enzinger FM and Weiss SW: Soft tissue tumors. 3rd edition. St Louis, C. V. Mosby, pp1067-1074, 1995.

50. Portera CA Jr, Ho V, Patel SR, Hunt KK, Feig BW, Respondek PM, Yasko AW, Benjamin RS, Pollock RE and Pisters PW: Alveolar soft part sarcoma: Clinical course and patterns of metastasis in 70 patients treated at a single institution. Cancer 91: 585-591, 2001.

51. Miettinen $\mathrm{M}$ and Ekfors T: Alveolar soft part sarcoma. Immunohistochemical evidence for muscle cell differentiation. Am J Clin Pathol 93: 32-38, 1990.

52. Rosai J, Dias P, Parham DM, Shapiro DN and Houghton P: MyoD1 protein expression in alveolar soft part sarcoma as confirmatory evidence of its skeletal muscle nature. Am J Surg Pathol 15: 974-981, 1991.

53. Foschini MP and Eusebi V: Alveolar soft-part sarcoma: A new type of rhabdomyosarcoma? Semin Diagn Pathol 11: 58-68, 1994.

54. Wang NP, Bacchi CE, Jiang JJ, McNutt MA and Gown AM: Does alveolar soft-part sarcoma exhibit skeletal muscle differentiation? An immunocytochemical and biochemical study of myogenic regulatory protein expression. Mod Pathol 9: 496-506, 1996.

55. Gómez JA, Amin MB, Ro JY, Linden MD, Lee MW and Zarbo RJ: Immunohistochemical profile of myogenin and MyoD1 does not support skeletal muscle lineage in alveolar soft part sarcoma. Arch Pathol Lab Med 123: 503-507, 1999.

56. Stockwin LH, Vistica DT, Kenney S, Schrump DS, Butcher DO, Raffeld $\mathrm{M}$ and Shoemaker RH: Gene expression profiling of alveolar soft-part sarcoma (ASPS). BMC Cancer 9: 22, 2009.

57. Kayton ML, Meyers P, Wexler LH, Gerald WL and LaQuaglia MP: Clinical presentation, treatment, and outcome of alveolar soft part sarcoma in children, adolescents, and young adults. J Pediatr Surg 41: 187-193, 2006.

58. Sherman N, Vavilala M, Pollock R, Romsdahl M and Jaffe N: Radiation therapy for alveolar soft-part sarcoma. Med Pediatr Oncol 22: 380-383, 1994.

59. Jia Y, Wu D, Shang C, Yu J and Zhang KR: Alveolar soft part sarcoma occurring on the abdominal wall of a 2-year-old child. J Pediatr Hematol Oncol 33: e80-e82, 2011.

60. Pappo AS, Parham DM, Cain A, Luo X, Bowman LC, Furman WL, Rao BN and Pratt CB: Alveolar soft part sarcoma in children and adolescents: Clinical features and outcome of 11 patients. Med Pediatr Oncol 26: 81-84, 1996.

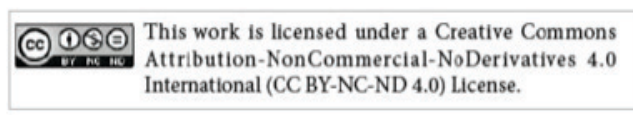

